\title{
Molecular Cloning, Modeling, and Characterization of Type 2 Metallothionein from Plantago ovata Forsk
}

\author{
Amitava Moulick, ${ }^{1}$ Debashis Mukhopadhyay, ${ }^{2}$ Shonima Talapatra, ${ }^{1}$ \\ Nirmalya Ghoshal, ${ }^{1}$ and Sarmistha Sen Raychaudhuri ${ }^{1}$ \\ ${ }^{1}$ Department of Biophysics, Molecular Biology and Bioinformatics, University of Calcutta, 92, APC Road, Kolkata 700009, India \\ ${ }^{2}$ Structural Genomics Section, Saha Institute of Nuclear Physics, 1/AF Bidhan Nagar, Kolkata 700 064, India \\ Correspondence should be addressed to Sarmistha Sen Raychaudhuri; sarmistha_rc@rediffmail.com
}

Received 28 September 2012; Revised 31 January 2013; Accepted 11 February 2013

Academic Editor: Alfredo Ciccodicola

Copyright (C 2013 Amitava Moulick et al. This is an open access article distributed under the Creative Commons Attribution License, which permits unrestricted use, distribution, and reproduction in any medium, provided the original work is properly cited.

Plantago ovata Forsk is a medicinally important plant. Metallothioneins are cysteine rich proteins involved in the detoxification of heavy metals. Molecular cloning and modeling of MT from $P$. ovata is not reported yet. The present investigation will describe the isolation, structure prediction, characterization, and expression under copper stress of type 2 metallothionein (MT2) from this species. The gene of the protein comprises three exons and two introns. The deduced protein sequence contains 81 amino acids with a calculated molecular weight of about $8.1 \mathrm{kDa}$ and a theoretical pI value of 4.77 . The transcript level of this protein was increased in response to copper stress. Homology modeling was used to construct a three-dimensional structure of $P$. ovata MT2. The 3D structure model of $P$. ovata MT2 will provide a significant clue for further structural and functional study of this protein.

\section{Introduction}

The seed husk of Plantago ovata Forsk has been used as a dietary fiber for a long time, and more recently it has been shown to reduce the development of endothelial dysfunction, hypertension, and obesity [1]. The plant has been shown also to reduce carbohydrate absorption and postprandial rise of glucose and insulin levels in type 2 diabetes patients [2]. It also lowers plasma lipids by altering hepatic and bile acid metabolism [3]. Studies on the effect of aqueous extracts of $P$. ovata in patients with diabetes found that it can reduce hyperglycemia via inhibition of intestinal glucose absorption and enhancement of motility [4].

Heavy metal ions play essential roles in many physiological processes. Among these, copper $(\mathrm{Cu})$ is an essential micronutrient in plants. It is a component of several electron transport enzymes and is involved in catalyzing the redox reactions in mitochondria and chloroplasts [5]. Copper also plays important roles in respiration, carbohydrate distribution, protein metabolism, water relations, reproduction, and disease resistance [6]. However, at high concentration, this metal can inhibit plant growth, causes degradation of chlorophyll, and impedes photosystem (PS) II activity [7, 8]. Soil can be naturally rich in heavy metals [6] which become the major pollutant by industrial processes, such as steel production and petroleum processing [9]. Copper is considered to be one of the most important pollutants of agricultural soils [6]. Sources of $\mathrm{Cu}$ contamination include mining and smelting; urban, industrial, and agricultural wastes; and the use of fungicides and herbicides [10]. A general mechanism of copper toxicity may be the generation of harmful reactive oxygen species (ROSs) which can damage biological molecules (DNA, RNA, and proteins) and membranes by inducing lipid peroxidation [5]. In response to toxic levels of heavy metals, plants evolved a suitable mechanism that controls the uptake and accumulation of both essential and nonessential heavy metals by synthesizing cysteine-rich, metal-binding peptides like metallothioneins which in turn helps in detoxification of heavy metals by chelation and sequestration in the vacuole $[11,12]$.

Metallothioneins (MTs) can protect cells against the toxic effects of copper by chelating heavy metal [13]. MTs are 
proteins rich in cysteine (Cys or $\mathrm{C}$ ) residues that bind heavy metals and play a role in buffering the intracellular concentration of free thiophilic metal ions, such as $\mathrm{Cu}, \mathrm{Zn}$, and Cd [14]. MTs are widely distributed among the animal and plant kingdom. MTs are low molecular weight proteins which contain two metal-binding, cysteine-rich domains linked by a Cys-free spacer which varies between plant and animal [12]. MTs also play important roles in regulation of metalloenzymes and transcription factors, scavenging of reactive oxygen species, metabolism of metallodrugs and alkylating agents, response to stress conditions, and apoptosis [15]. Classically, MTs were grouped into three classes according to their sequence similarities [12]. Afterward, it was classified into 15 families on the basis of taxonomic relationships, with plant MTs being placed into Family 15 [16]. All plant MTs (class II) were further distributed into four types, according to the distribution of cysteine residues in the amino- and carboxy-terminal regions similarities. The spacer region separating these domains in Type 2 MTs is much more variable between species [12]. In the present study, we found that amino acid sequence of Plantago ovata MT2 is very similar to Plantago major MT2, and the only difference between them was in the spacer region. Several plant MT proteins are found in the data base but very little experimental structural information has yet been reported so far due to difficulties in protein purification. The structure is very useful to understand the biological features and functions of a protein. Therefore, theoretical method is needed to predict the three-dimensional structure of proteins.

A novel finding of metallothionein type 2 gene from Plantago ovata is reported here. The objective of the present work is to investigate the MT2 expression pattern against copper toxicity. The isolated and molecular characterized MT2 transcript and its predicted structure contribute to a better understanding of MT2 on the basis of structurefunction relationship in an important medicinal plant like $P$. ovata.

\section{Materials and Method}

2.1. Plant Material and Treatment. Seeds of Plantago ovata were collected from Gujarat, India. The seeds were sterilized in $10 \%$ sodium hypochlorite and washed five times to wash off the excess bleach totally from the seed surface and imbibed in sterilized distilled water. Next day, the seeds were transferred to agar-sucrose media containing $3 \%(\mathrm{w} / \mathrm{v})$ sucrose (SRL, Mumbai, India) and $0.9 \%(w / v)$ agar (SRL, Mumbai, India) for germination according to the method as described by Das and Sen Raychaudhuri [17]. Eight-dayold seedlings were transferred to liquid Murashige and Skoog (MS) medium (Himedia, Mumbai, India) supplemented with different concentrations of $\mathrm{CuCl}_{2}(20 \mu \mathrm{M}, 40 \mu \mathrm{M}$, and $80 \mu \mathrm{M})$ along with control. The specified doses have been selected after considering the $\mathrm{LD}_{50}$ of copper in P. ovata. The $\mathrm{LD}_{50}$ of copper was found to be $120 \mu \mathrm{M}$ in case of $P$. ovate, and hence, a range of lower doses have been selected [18]. $\mathrm{CuCl}_{2}$ (copper (II) Chloride) (Merck, Darmstadt, Germany) treatment was carried out for $24 \mathrm{~h}$ and $72 \mathrm{~h}$ to observe the changes of MT transcript levels.

2.2. Genomic DNA Extraction. Genomic DNA was isolated from seedlings following the standard protocol of Edwards et al. [19]. Seedlings were crushed gently using DNA extraction buffer followed by a centrifugation to remove the tissue fragments. Supernatant was taken, and equal volume of phenol, chloroform (1:1) (SRL, Mumbai, India), was added to it. After that a centrifugation was carried out, and the supernatant was washed with equal volume of chloroform. Finally the clear upper aqueous phase was collected, and $3 \mathrm{M}$ ammonium acetate and equal volume of isopropanol (SRL, Mumbai, India) were added and mixed. DNA was spooled with glass capillary and washed with $70 \%$ ethanol (SRL, Mumbai, India). Subsequently, the DNA was air dried and dissolved in sterile triple distilled water. The DNA was run in $1 \%$ agarose gel and spectrophotometrically scanned to check the quality and quantity.

2.3. Total RNA Extraction. Total RNA was extracted from seedlings using RNeasy Plant Mini Kit (Qiagen, Hamburg, Germany) according to the manufacturer's protocol. All the glass goods were treated with diethyl pyrocarbonate (Sigma, St. Louis, USA) prior to RNA extraction. RNA was quantified spectrophotometrically. The extracted RNA was preserved at $-70^{\circ} \mathrm{C}$.

2.4. Primer Designing. The primers for $P$. ovata MT2 were designed based on published sequences. Published sequences were taken using Blast search, and they were aligned using ClustalW. Primers were designed from those regions of Plantago major MT2 sequence showing best homology with other published sequences. The forward and reverse primers of MT2 were of 23 and 22 bases, respectively. The primers were designed in such a way so that their melting temperature would be close. The sequences of forward and reverse MT2 primers were $5^{\prime}$-TCTTGCTGCAACGGAAACTGTGG-3' and $5^{\prime}$-AGTTGTCACCGCACTTGCACCC- ${ }^{\prime}$, respectively. Ubiquitin was taken as a housekeeping gene $[20,21]$ to normalize the reverse transcription PCR analysis. The sequences of ubiquitin primers were designed in the same procedure as described above. The sequences of forward and reverse primer of $P$. ovata ubiquitin were $5^{\prime}$-TGAAAACTTTTACAGGCAAGACC- $3^{\prime}$ and $5^{\prime}$-GACGGAGTACCAAATGGAGAGTG-3' ${ }^{\prime}$, respectively.

\subsection{Polymerase Chain Reaction (PCR) and Reverse Transcrip-} tion Polymerase Chain Reaction (RT-PCR). PCR and RTPCR were carried out using designed primers. Taq enzyme, Taq buffer, and $\mathrm{MgCl}_{2}$ (magnesium chloride) used in PCR were brought from Genei (Bangalore, India) and were used according to the manufacturer's protocol. The thermal profile for PCR amplification of MT2 was as follows: $94^{\circ} \mathrm{C}$ for $2 \mathrm{~min}$ followed by 35 cycles at $94^{\circ} \mathrm{C}$ for $1 \mathrm{~min}, 62^{\circ} \mathrm{C}$ for $30 \mathrm{sec}$, and $72^{\circ} \mathrm{C}$ for $1 \mathrm{~min} 30 \mathrm{sec}$ and a final extension at $72^{\circ} \mathrm{C}$ for $10 \mathrm{~min}$. RT-PCR was carried out using One Step RT-PCR Kit (Qiagen, Hamburg, Germany) according to 
the manufacturer's protocol with the designed primers. The reverse transcription experiments were carried out using equal amounts of total RNA ( $2 \mu \mathrm{g})$ extracted from copper stressed and control seedlings. Simultaneously, RT-PCR was also carried out for ubiquitin in the same conditions of experiment. This step was essential to estimate the efficiency of reverse transcription system after 25 cycles of amplification. PCR and RT-PCR was carried out using GeneAmp PCR System (Applied Biosystems, Carlsbad, USA). The thermal profile for RT-PCR amplification of MT2 was as follows $50^{\circ} \mathrm{C}$ for $30 \mathrm{~min}, 95^{\circ} \mathrm{C}$ for $15 \mathrm{~min}$ followed by 25 cycles at $94^{\circ} \mathrm{C}$ for $1 \mathrm{~min}, 62^{\circ} \mathrm{C}$ for $30 \mathrm{sec}$ and $72^{\circ} \mathrm{C}$ for $1 \mathrm{~min} 30 \mathrm{sec}$ and a final extension at $72^{\circ} \mathrm{C}$ for $10 \mathrm{~min}$. The RT-PCR cycle for ubiquitin was: $50^{\circ} \mathrm{C}$ for $30 \mathrm{~min}, 95^{\circ} \mathrm{C}$ for $15 \mathrm{~min}$ followed by 25 cycles at $94^{\circ} \mathrm{C}$ for $1 \mathrm{~min}, 57^{\circ} \mathrm{C}$ for $1 \mathrm{~min}$, and $72^{\circ} \mathrm{C}$ for $1 \mathrm{~min} 30 \mathrm{sec}$ and a final extension at $72^{\circ} \mathrm{C}$ for $10 \mathrm{~min}$. Aliquots of $25 \mu \mathrm{L}$ of the PCR and RT-PCR products were run on $1.5 \%$ and $2 \%$ agarose gel, respectively. The gels were stained with ethidium bromide, and photographs were taken using a Gel Documentation System (BioRad, Hercules, USA). All RT-PCR reactions were replicated at least thrice from three independent RNA preparations. The pixel intensity of the band in gel was quantified using ImageJ software. The obtained results were subjected to statistical analysis using Kyplot and Microsoft Excel. All data were expressed as mean \pm standard error (SE). These data were analyzed by an analysis of variance (ANOVA). Group means were compared using Student's $t$ test if significance was found in ANOVA. Differences of the data were considered significant when $P \leq$ 0.05 .

2.6. Cloning and Sequence Analysis. Cloning and Sequence Analysis of The PCR product of metallothionein type 2 was purified by PCR clean-up kit (Chromus Biotech, Bengaluru, India) according to the manufacturer's protocol. The Metallothionein gene fragment was cloned into the vector pTZ57R/T using the protocol provided in the InsTAclone PCR cloning Kit (Fermentas, St. Leon-Rot, Germany). E. coli XL 1- blue was used as a host strain. After cloning the purified plasmid, it was sequenced using universal primers. The primer sequences are $5^{\prime}$-GTAAAACGACGGCCAGT$3^{\prime}$ and $5^{\prime}$-CAGGAAACAGC- TATGAC- $3^{\prime}$. In case of ubiquitin, the PCR product was sequenced using gene specific primers. The sequences were analyzed using several programs such as BLASTN, BLASTX, BLASTP, and ClustalW $[22,23]$. The deduced amino acid sequence of $P$. ovata MT2 was analyzed to detect the N-terminal sorting signal using iPSORT (http://ipsort.hgc.jp/), and to determine the pI value and molecular weight using ExPASy (http://www.expasy.ch/tools/) [22]. To determine the hydrophobic nature of the amino acids in the protein, a hydropathy plot was created using ExPASy-ProtScale (http:// web.expasy.org/protscale/) choosing Kyte \& Doolittle scale, default parameters and window size 9. The disulfide bond partners in the $P$. ovata MT2 were predicted using DiANNA server (http://clavius.bc.edu/ clotelab/ DiANNA/main.html). For better estimation of the relationships between $P$. ovata MT2 and other reported
MTs, a phylogenetic tree was generated using Phylip 3.69 package (http://evolution.genetics.washington.edu/ phylip.html) enabling 100 bootstraps. Unique metallothionein sequences were selected from UniProt/Swiss-Prot knowledgebase (http://www.uniprot.org/docs/metallo. txt) $[24,25]$. One sequence from each metallothionein subdivision was taken along with all plant MT (Family 15) to generate a significant phylogenetic tree. As primers were designed from the available closest species Plantago major, amino acid sequences of metallothionein of this species were included in the phylogenetic tree. Sequence file (Phylip format) was run using seqboot choosing 100 replicate and all other default parameters. The outfile of this program was run using protpars for parsimony based phylogenetic analysis keeping the number of jumbles set to 10 and all other parameters as default, the output tree of this program was run using consense. Finally the unrooted tree was constructed using njplot (http://pbil.univ-lyon1.fr/software/njplot.html).

2.7. Expression Analysis Using Real-Time PCR. For metallothionein type 2 gene expression analysis, first strand cDNA was synthesized from total RNA $(\sim 2 \mu \mathrm{g})$ of all the treated (for $72 \mathrm{~h}$ with different concentrations of $\mathrm{CuCl}_{2}$ ) samples using high-capacity RNA-to-cDNA kit (Applied Biosystems, USA) according to the manufacturer's instruction. Real-time PCR was carried out in Step One Plus Real-Time PCR thermocycler (Applied Biosystems, UK). Each reaction contained 2X Power Cyber Green PCR master mix (Applied Biosystems, $\mathrm{UK}$ ), diluted cDNA, and $10 \mathrm{pmol}$ gene specific primers. The sequences of forward and reverse primers used in realtime PCR were $5^{\prime}$-TCTTGCTGCAACGGAAACTGTGG-3' and $5^{\prime}$-AGTTGTCACCGCACTTGCACCC-3', respectively. Following thermal conditions have been used: heating at $95^{\circ} \mathrm{C}$ for $10 \mathrm{~min}, 40$ cycles of denaturation at $95^{\circ} \mathrm{C}$ for $15 \mathrm{sec}$, annealing and extension at $60^{\circ} \mathrm{C}$ for $1 \mathrm{~min}$ followed by a melt curve analysis to ensure the amplification of only the desired amplicon. A gene encoding P. ovata actin was used as endogenous control. The primers used to amplify actin were $5^{\prime}$-ATCATGAAGTGTGATGTTGA-3' (forward) and $5^{\prime}$-ACCTTAATCTTCATGCTGCC- $3^{\prime}$ (reverse). The relative gene expression was conducted using the $2^{-\Delta \Delta \mathrm{CT}}$ method [26]. The data of relative gene expression were analyzed by Step One software version 2.1 (Applied Biosystems, UK). All the experiments were performed in triplicate. A negative control was included in each reaction. The results presented here are means of three replicates, and the bars indicate standard deviation. Statistical significance of real-time experiment results were analyzed by analysis of variance (ANOVA) using Kyplot software.

2.8. Molecular Modeling of P. ovata MT2. The deduced amino acid sequence of $P$. ovata MT2 (complete coding region) was submitted to ModWeb server (https://modbase.compbio.ucsf.edu/scgi/modweb.cgi) for comparative protein structure modeling choosing default parameters [27]. ModWeb performs automated comparative modeling which relies on PSI-BLAST, IMPALA, and MODELLER [28]. This server uses ModPipe (version SVN.r1340:1348M) 
PoMT2 gene (partial CDS):

1 TCT TGC TGC AAC GGA AAC TGT GGA TGT GGA TCT GGC TGC AAG TGC

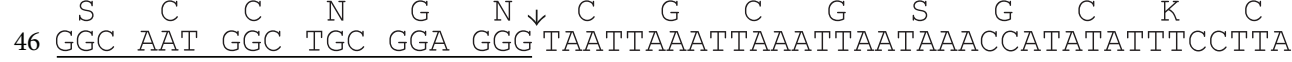
$\begin{array}{lllllllllll} & \mathrm{N} & \mathrm{N} & \mathrm{C} & \mathrm{G} & \mathrm{G}\end{array}$

100 ATtACTCAATTCTGTAAATTAGCTAATAGCTAGgGTTGTATTGATTTGGTACATTTTTA

159 TTTCAGATCTGGGTTTGATTCTCCATCTCTTGGTATTTAAAAATGATGATGTTAATTTG

$218 \mathrm{CAGG}^{\star}$ TGC AAG ATG TAC CCC GAT TTG AGC TAC TCT GAG GCC ACC GTG

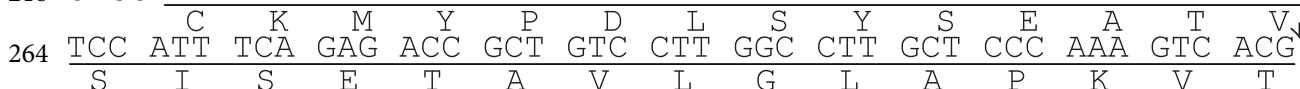
309 TATGCATTTTTCTAATAATCCGATTTATTTCATCGTTGTTTTGTTAGATATACTTATG

368 AGATTCATAATTAATTTAAGTTAGATAACCTTATCAACTTAACAGCAATTATAGATCAT

427 GTGATCCCTAAATTTATGCCCTGATTTTTTAATATTGCATAGAGTAACAATTAGTGGTA

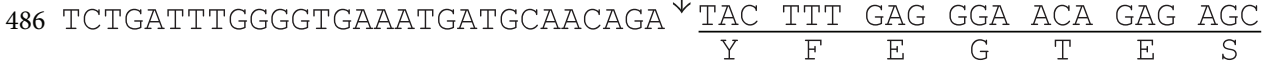
\begin{tabular}{ccccccccccccccc}
536 & GTT & GTT & GCT & GGG & TCC & GAG & AAC & GGG & TGC & AAG & TGC & GGT & GAC & AAC \\
\hline V & V & A & G & S & E & N & G & C & K & C & G & D & N
\end{tabular}

(a)

PoMT2 mRNA (complete CDS):

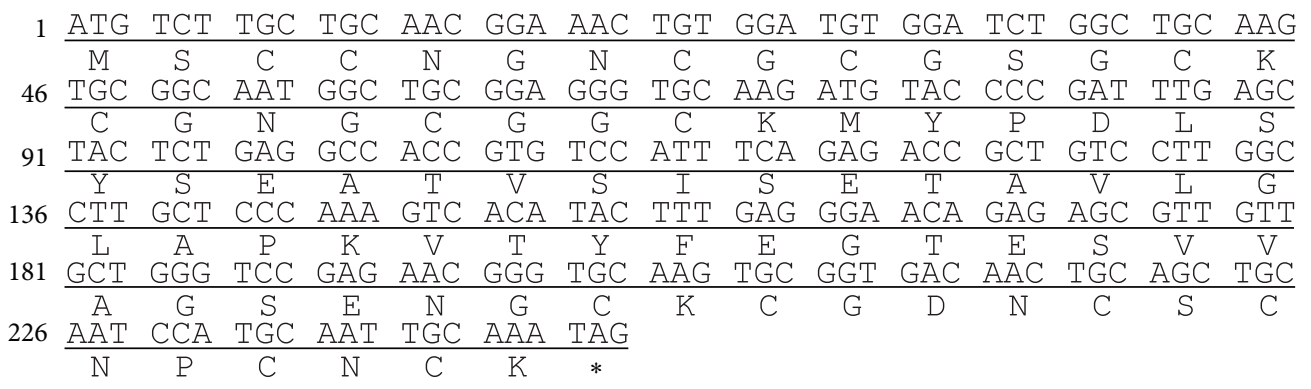

(b)

FIgURE 1: Nucleotide and deduced amino acid sequences of PoMT2. Two introns interrupt the coding region at the points marked by arrows. (a) Partial coding region encodes a protein of 71 amino acids (GenBank Accession no. GU596501 and GU596503). (b) Complete coding region encodes a protein of 81 amino acids.

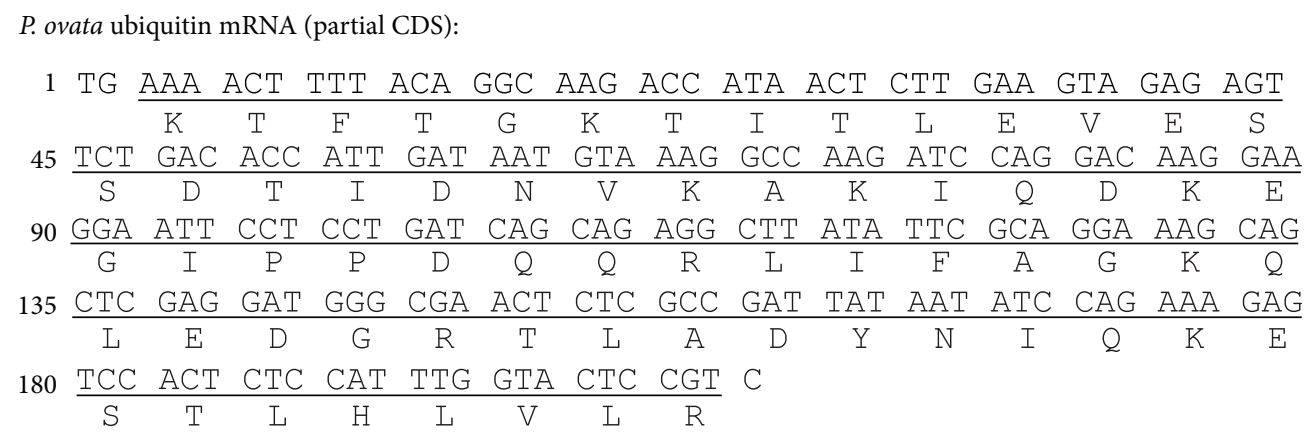

FIGURE 2: Nucleotide and deduced amino acid sequences of $P$. ovata ubiquitin (GenBank Accession no. JQ419758). Partial coding region encodes a protein of 67 amino acids.

as underlying software pipeline. Human ADAM22, chain A (Protein Data Bank ID 3G5C) was used as template structure (template region 447-523) for modeling of $P$. ovata MT2. The structure was reconstructed using the DeepView/Swiss-PdbViewer3.7 [15].

\section{Result and Discussion}

3.1. Cloning and Sequence Analysis. PCR was carried out using genomic DNA, and a $578 \mathrm{bp}$ fragment was found. This fragment was purified, cloned, and sequenced (Figure 1(a)). On the other hand, RT-PCR was carried out using total RNA, and a $214 \mathrm{bp}$ fragment was found which was also purified, cloned, and sequenced (Figure 1(a)). Comparing both the sequences, it was found that the type 2 metallothionein gene of $P$. ovata has 3 exons $(0-63,222-308$, and 515-577) and 2 introns (64-221 and 309-514) in its sequence. MT2 of some other plant species also show 3 exons and 2 introns like Helianthus annuus (GenBank accession no. EF431954), Typha angustifolia (GenBank accession no. 
$24 \mathrm{~h}$

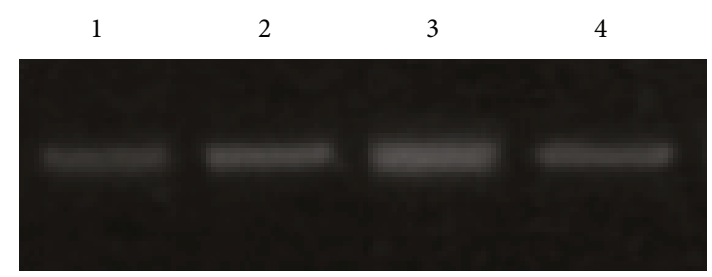

(a)

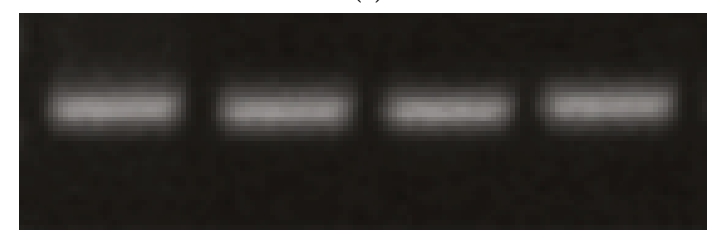

(b)

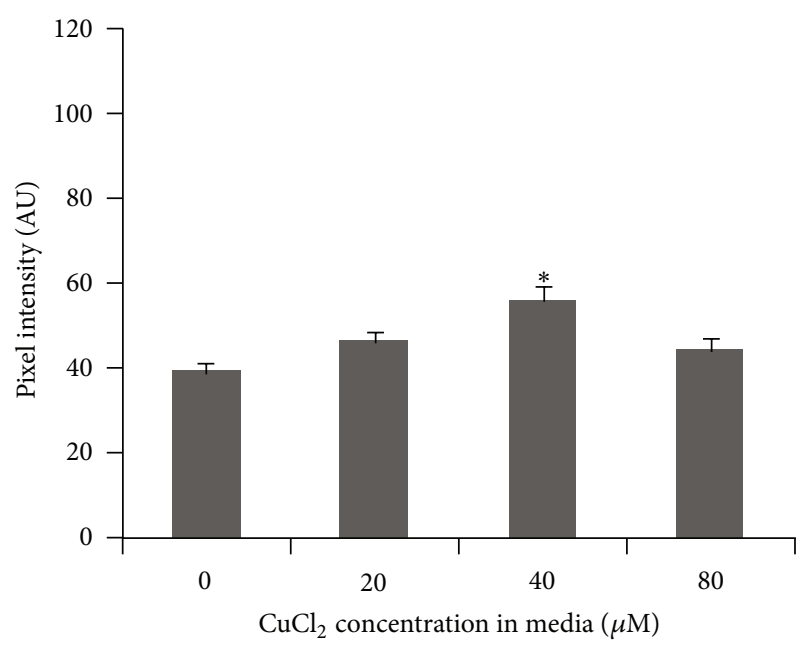

$72 \mathrm{~h}$

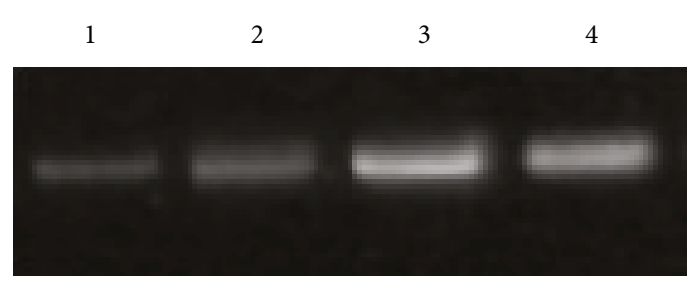

(c)

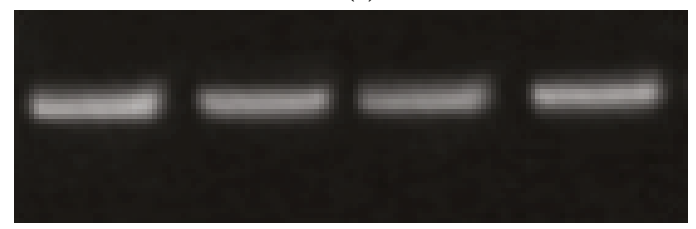

(d)

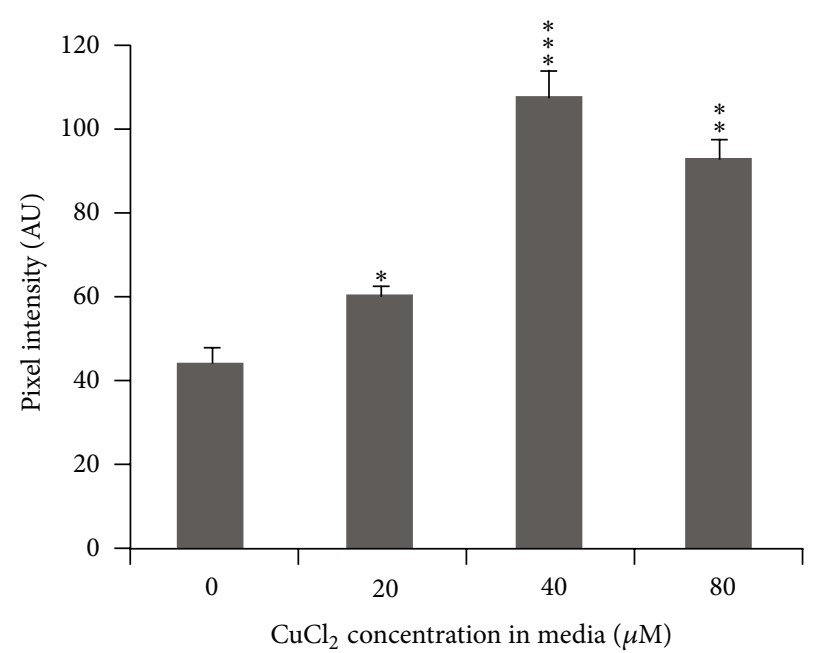

(e)

FIGURE 3: Results from reverse transcription PCR analysis. Upper panel showing RT-PCR band in agarose gel of metallothionein type 2 $((\mathrm{a}),(\mathrm{c}))$ and ubiquitin ((b),(d)) transcripts after treatment with different concentrations of $\mathrm{CuCl}_{2}(0,20,40$, and $80 \mu \mathrm{M}$ in lane no. $1,2,3$, and 4 , resp.). Lower panel showing densitometry graph of corresponding band of MT2. Data represent the mean \pm SE. Asterisks indicate significant differences at $P<0.05\left({ }^{*}\right), P<0.01\left({ }^{* *}\right)$, or $P<0.001\left(^{* * *}\right)$ compared to respective controls. AU means arbitrary unit.

EF543085), and Hordeum vulgare (GenBank accession no. JN997433).

After splicing out of the introns, $P$. ovata forms a $214 \mathrm{bp}$ MT2 mRNA. This sequence was searched for similar other sequences with the help of BLAST tool and many other sequences of metallothionein type 2 were found with 75$90 \%$ of sequence identity, for example, Plantago major MT2, Avicennia marina MT2, and Ilex paraguariensis (GenBank accession numbers are AJ843994, AF333385, and JX271039, resp.). The deduced amino acid sequence was also searched by BLASTp tool to confirm that the obtained sequence was of metallothionein type 2 . The resultant nucleotide sequence was aligned with some other reported metallothionein type 2 with the help of ClustalW, and similarity was found. BLASTp analysis of the deduced amino acid sequence also showed that the fragment conformed to metallothionein type 2 super family. Finally, it was confirmed that the cloned sequence was of $P$. ovata metallothionein type 2 . The successfully isolated partial coding regions of MT2 gene and mRNA sequences of $P$. ovata were submitted to GenBank with the accession numbers GU596501 and GU596503, respectively, and these were the first sequences of metallothionein from this species. A later study by our group has sequenced the complete coding regions of MT2 from $P$. ovata (PoMT2) (Figure 1(b)) (submitted to GenBank having accession no. KC414846) encoding an 81-amino-acid protein with a calculated molecular weight of about $8.1 \mathrm{kDa}$ and a theoretical $\mathrm{pI}$ value of 4.77. The primer sequences used here were $5^{\prime}$-ATGTCTTGCTGCAACGGAAACT- $3^{\prime}$ (forward) and $5^{\prime}$ CTATTTGCAATTGCATGGATTG- ${ }^{\prime}$ (reverse). The thermal profile for RT-PCR reaction was as follows: $50^{\circ} \mathrm{C}$ for $30 \mathrm{~min}, 95^{\circ} \mathrm{C}$ for $15 \mathrm{~min}$ followed by 25 cycles at $94^{\circ} \mathrm{C}$ for $1 \mathrm{~min}, 54^{\circ} \mathrm{C}$ for $30 \mathrm{sec}$, and $72^{\circ} \mathrm{C}$ for $1 \mathrm{~min} 30 \mathrm{sec}$ and a final extension at $72^{\circ} \mathrm{C}$ for $10 \mathrm{~min}$. Analysis of this PoMT2 


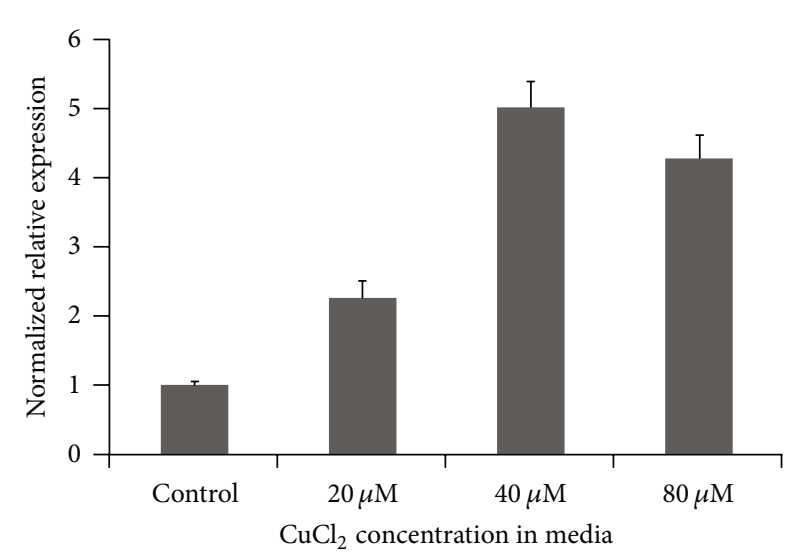

FIgURE 4: Real-time PCR expression profile of PoMT2 normalized to the housekeeping gene actin under $0,20,40$, and $80 \mu \mathrm{M}$ copper stress. The results indicate means of three replicates with their standard deviation.

using iPSORT predicted that it does not have any mitochondrial targeting peptide or chloroplast transit peptide. Using DiANNA server, it was found that the PoMT2 has 7 predicted disulfide bond between 3-8, 4-75, 10-67, 14-20, 16-78, 23-80, and 69-73 amino acid positions. The terminal domain contained $0 \%$ aromatic, $26.3 \%$ nonpolar aliphatic, $60.5 \%$ polar, and $10.5 \%$ charged amino acids. In case of spacer region, these values were $9.3 \%, 41.9 \%, 30.2 \%$ and $18.6 \%$ respectively. This protein sequence of PoMT2 was compared using ClustalW and Blastp indicates that PoMT2 has 84\% sequence identity to the MT2 encoded by Plantago major followed by Avicennia marina $72 \%$ identity, and Citrullus lanatus at $70 \%$. It has been reported that class I MTs contain 20 highly conserved $\mathrm{C}$ residues showing homology to mammalian MTs whereas MTs without this strict arrangement of cysteines are referred to as class II MTs. These MTs do not show homology to mammalian MTs. Type 2 MTs contain CC, CXC, and CXXC motifs in the N-terminal and CXC motif in the C-terminal domain. X can be any amino acid other than cysteine. It also contains a CGGC motif at the end of the N-terminal cysteinerich domain and a spacer of approximately 40 amino acid residues $[12,15]$. In the present experiment, PoMT2 follows all these criteria confirming that the isolated metallothionein is a class II Type 2 plant metallothionein.

The reverse transcription PCR product for ubiquitin was sequenced. A 204 bp sequence was obtained (Figure 2) (encoding 67 amino acids) which was searched for similar other sequences with the help of BLAST tool as described above. It was found that the cloned sequence showed a good similarity with some other published ubiquitin sequences. For further confirmation, the deduced amino acid sequence was also searched by BLASTp tool, and $99 \%$ sequence identity was found with other reported ubiquitin sequences of Arabidopsis thaliana, Plantago major, Musa acuminate, and Medicago truncatula (GenBank accession numbers are AAB95251, CAH56488, AAQ07454, and AET01627, resp.). Finally, it was submitted to GenBank with the accession number JQ419758. It was the first ubiquitin sequence of $P$. ovata.

\subsection{Expression of PoMT2 Transcript in Different Stress Condi-} tion. To characterize PoMT2 transcript accumulation, total RNA extracted from various stress conditions were subjected to reverse transcription (RT)-PCR reaction. The seedlings were treated with $0,20,40$, and $80 \mu \mathrm{M}$ of $\mathrm{CuCl}_{2}$ solutions for $24 \mathrm{~h}$ and $72 \mathrm{~h}$ (Figure 3). In each case, 25 cycles of PCR reaction with first strand cDNA was carried out. All the reactions showed the presence of a $214 \mathrm{bp}$ band. The intensity of the band was maximum in $40 \mathrm{M} \mathrm{CuCl}_{2}$ treated sample (after $24 \mathrm{~h}$ ) which was $42.5 \%$ higher as compared to control. PoMT2 expression level was upregulated by all the three concentrations of $\mathrm{CuCl}_{2}$ after $72 \mathrm{~h}$. The expression of PoMT2 was found to be higher in $40 \mu \mathrm{M}(144.6 \%)$ and $80 \mu \mathrm{M}$ (111\%) treated samples than control. In our experiment, we have compared the expression level of MT2 with that of ubiquitin under similar experimental conditions. The result indicated an enhancement of MT type 2 expression with increasing doses of copper treatment.

Real-time PCR was used for more precise analysis of expression pattern of metallothionein type 2 gene. The results of real-time PCR clearly indicated stress induced accumulation of MT2 transcript (Figure 4). At lower doses, the expression of MT2 slightly increased with respect to control but at higher doses a marked increase in expression has been observed. Highest expression (4.9-fold) was found in $40 \mu \mathrm{M}$ treated sample. However, in $80 \mu \mathrm{M}$ treated sample MT2 expression was decreased slightly than $40 \mu \mathrm{M}$ dose but still remained higher (4.2-fold) than control. These results are clearly in agreement with the data obtained in reverse transcription PCR. Almost similar expression pattern was reported for MT2 in Arabidopsis [18]. In this species, MT2a mRNA was induced strongly whereas MT2b mRNA levels increased only slightly upon exposure to copper [29]. In another experiment, Physcomitrella patens MT2 transcript was also induced by copper stress [30]. Significant increase in the MT2 transcripts level of Bruguiera gymnorrhiza was also found in response to heavy metals like zinc, copper and lead [31]. The current data suggest that higher doses of copper stress upregulate MT2 expression in plant which in turn results in a protection system against this metal.

3.3. Structure Prediction and Phylogenetic Analysis. Homology modeling was used to construct PoMT2 structure. This protein has three significant regions. The N-terminal (residues 1-23) and C-terminal (residues 67-81) Cys rich metal binding domains were fairly conserved with other sequences whereas the spacer region (residues 24-66) was variable. These three regions of PoMT2 were modeled together using ModWeb server. As the protein data bank did not contain any plant MT structure with a sequence that would be sufficiently similar to the complete sequence of this protein, human ADAM22 (Protein Data Bank ID $3 \mathrm{G} 5 \mathrm{C})$ was used as template structure. 3D structure was made with $E$-value of 0.015 and $25.00 \%$ sequence identity. The final structure was shown with RMSD value $12.227 \AA$ 


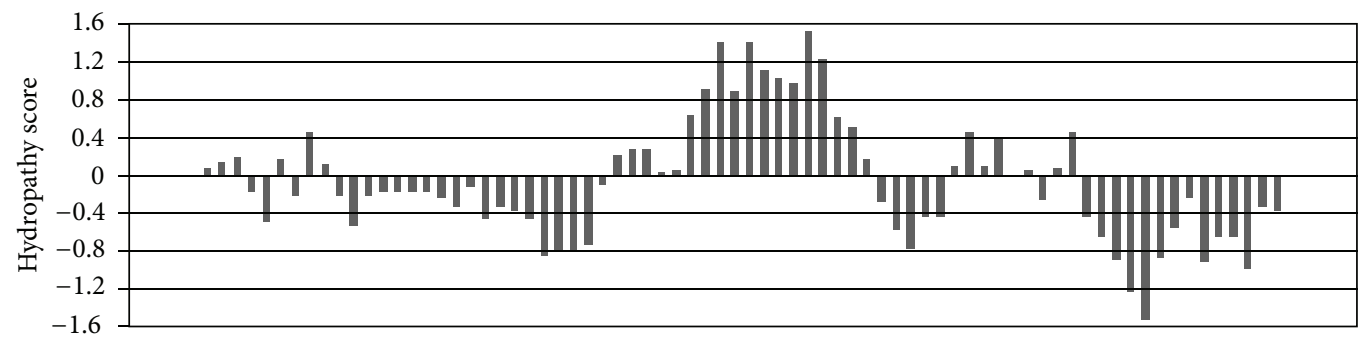

P. major MT2 P. ovata MT2

A. deliciosa MT2

M. domestica MT2

T. repens MT2

V. faba MT2

C. arietinum MT2

R. communis MT2

S. lycopersicum MT2B

N. glutinosa MT2

B. juncea MT22

B. rapa MT2

B. rapa pekinensis MT2

A. thaliana MT2A

B. juncea MT21

B. juncea MT25

B. juncea MT23

A. thaliana MT2B

C. arabica MT1
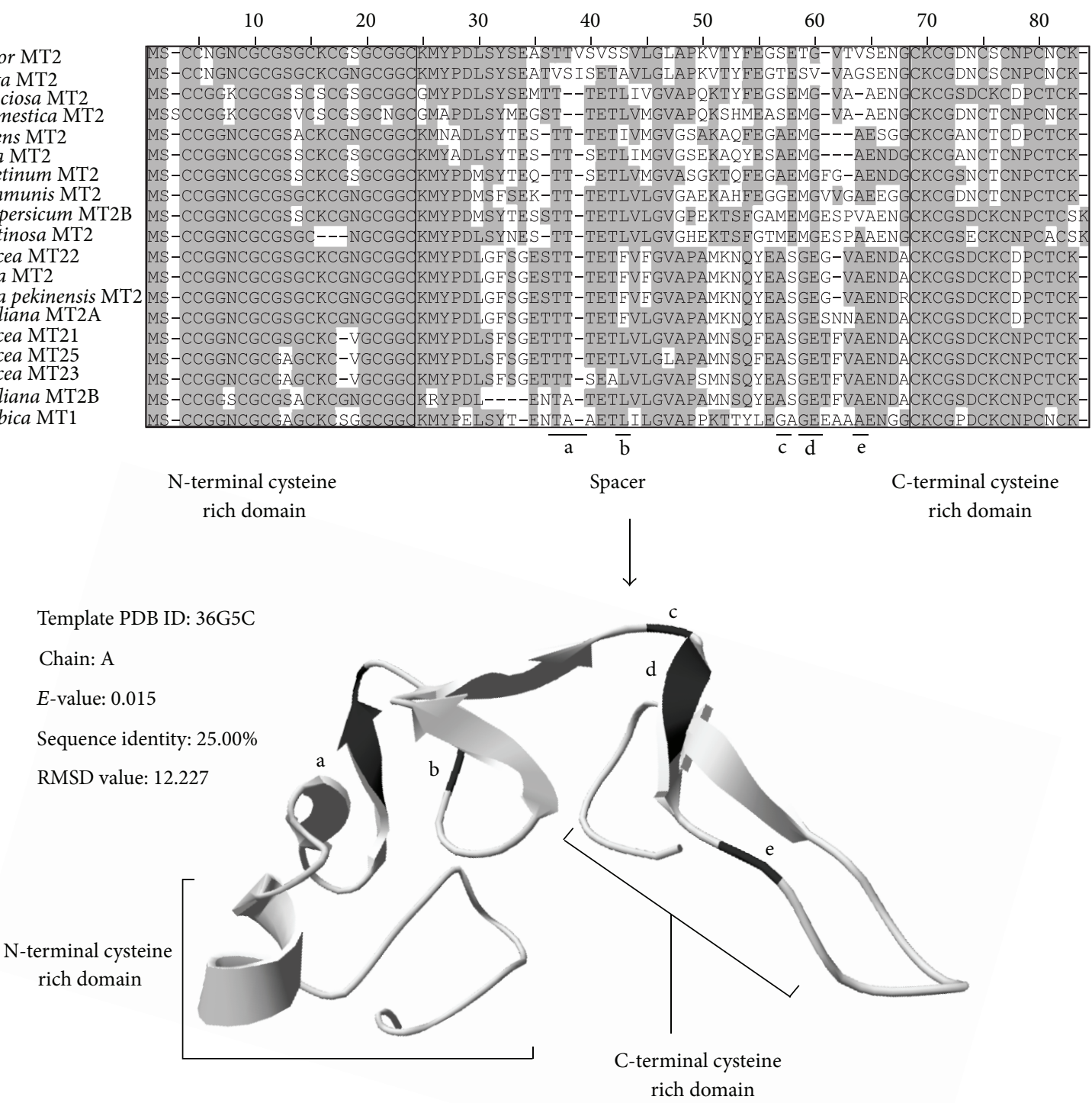

Figure 5: Modeling and analysis of PoMT2. Five distinct motifs of PoMT2 are indicated as a, b, c, d, and e and colored black on the 3D model. Hydropathy scores of each amino acid (position 5-77) of PoMT2 are shown at corresponding regions. Multiple sequence alignment (shading showed residues identical for the given position in most sequences) showing relationship between PoMT2 and other plant MTs including Plantago major MT2, Solanum lycopersicum MT2B, Nicotiana glutinosa MT2, Actinidia deliciosa MT2, Malus domestica MT2, Coffea arabica MT1, Arabidopsis thaliana MT2A, Brassica juncea MT22, Brassica rapa subsp. pekinensis MT2, Brassica rapa MT2, Arabidopsis thaliana MT2B, Brassica juncea MT21, Brassica juncea MT25, Brassica juncea MT23, Ricinus communis MT2, Cicer arietinum MT2, Trifolium repens MT2, and Vicia faba MT2 (Uniprot IDs are Q5ZF75, Q40158, Q40396, P43390, O24058, P43396, P25860, P69163, Q39269, P69164, Q38805, P56168, P56172, P56170, P30564, Q39459, P43398, and Q41657, resp.). 


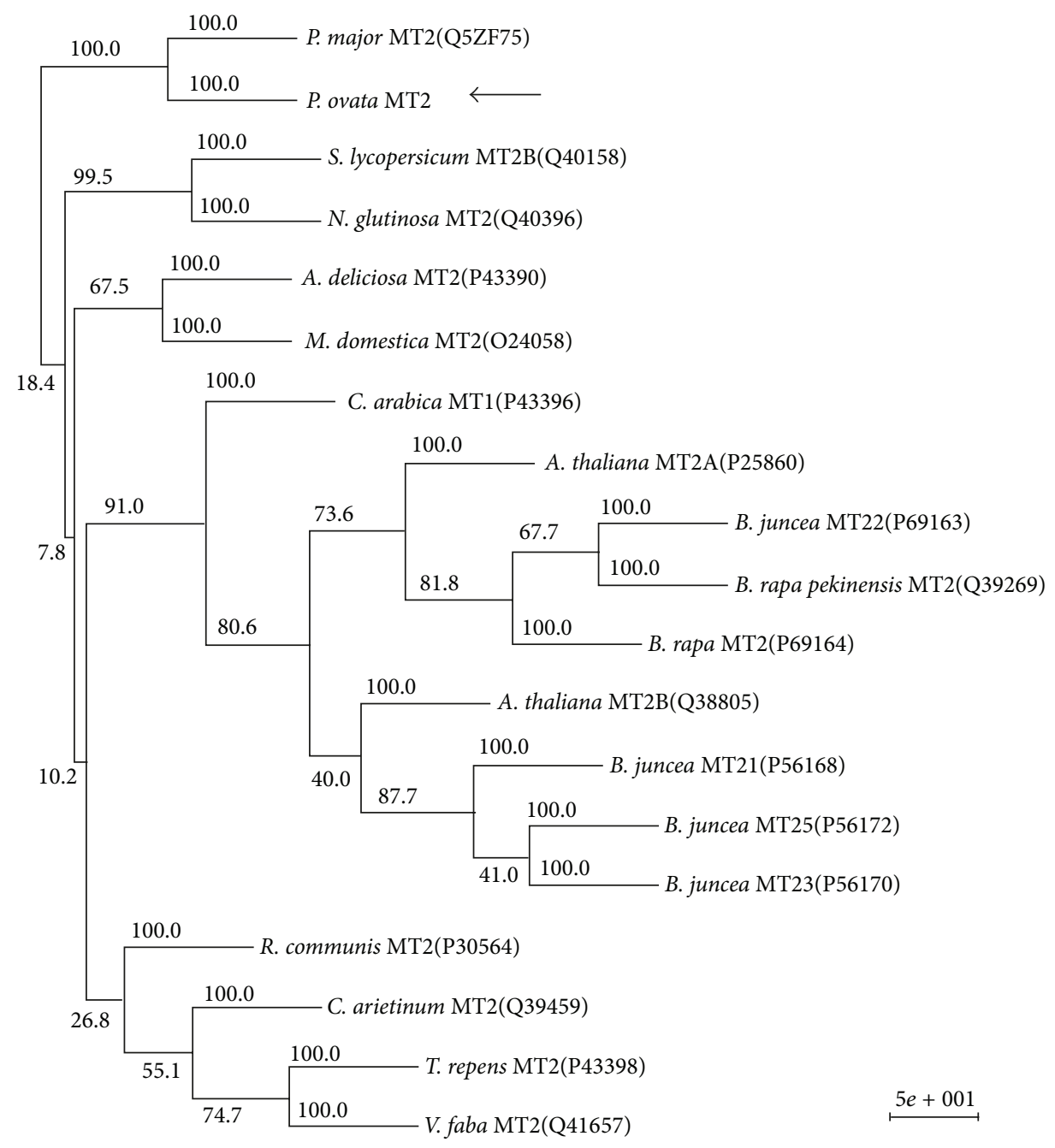

FIgure 6: Phylogenetic tree of PoMT2 with other unique MTs selected from UniProt/Swiss-Prot knowledgebase. This figure showed only the clade where PoMT2 belong with an arrow indicating P. ovata MT2. The phylogenetic tree depicts interrelationship of different MT2 proteins collected from different species, shown as operational taxonomic units. The Uniprot IDs for each protein is given in parenthesis with the percentage bootstrap values along the branch length.

with respect to the template. The resulting structures of $\mathrm{N}$ and C-terminal Cys rich domain showed a single $\alpha$-helix and $\beta$-sheet, respectively, whereas spacer region showed five $\beta$-sheets (46.5\% of spacer) at the core of the protein. A similar structure was found in the spacer region of Triticum durum [15]. According to the hydropathy plot (Figure 5) of PoMT2 most of the amino acids residing in spacer region showed high score for hydrophobicity whereas the score was overall low for C-terminal amino acid residues. Leucine (at 46 position) showed the highest score of +1.522 whereas Lysine (at position 68) showed the lowest score of -1.522 .

A phylogenetic tree (see Supplementary Figure in Supplementary Material available online at http://dx.doi .org/10.1155/2013/756983) was constructed using Phylip 3.69 package enabling 100 bootstraps. Only the important (for $P$. ovata MT2) cluster of total phylogenetic tree is shown in Figure 6 . The phylogenetic analysis suggested that PoMT2 was highly similar to MT2 of P. major. These two MT2s might originate from a common evolutionary ancestry. These two proteins mainly differ with respect to the sequence of amino acids in the spacer region. The closest neighbor of $P$. ovata MT2 happened to be MT2 of Solanum lycopersicum. Multiple sequence alignment (Figure 5) of PoMT2 with other plant MT2s supported this data. It revealed that both the terminal metal binding regions were highly conserved in most of the species, and the spacer regions varied between species. The hydropathy plot indicated that the spacer formed the core scaffold of the proteins. Basically, the lack of sequence identity of this spacer region reflected different structural motifs for the same protein in different plants, whereas the $\mathrm{N}$ - and C-terminal domains are conserved indicating their functional importance. Having different conformations but similar functionality, which is apparent from our phylogenetic analysis (Figure 6), would indicate that the same class of 
protein would behave differentially under different stress conditions, which would be guided by their $3 \mathrm{D}$-conformations. In PoMT2, at least five motifs could be identified which are distinct in terms of the nature of the amino acids and would likely contribute to a novel fold of the protein. This behavioral difference was reported in Polulus alba where it showed that MT2 transcript level was decreased with increasing time of copper treatment [32].

\section{Conclusion}

A class II type 2 MT gene, designated as PoMT2, was successfully isolated from Plantago ovata. The sequence of this protein showed similarity with other reported MT2s. Molecular modeling and characterization of this protein were carried out. The distinct amino acid sequences of $P$. ovata belonged to the spacer region. Like other plant MT2s, PoMT2 was found to be upregulated under copper stress as a defense of the plant system. These data suggest that the spacer region is not involved in heavy metal detoxification but it has an evolutionary significance. Since detailed knowledge of the protein structure is required to know its biological functions, the predicted three-dimensional structures of PoMT2 will certainly smooth the way to understand the guidelines for future experiments.

\section{Acknowledgment}

The authors express sincere gratitude to University Grants Commission (UGC) for the award of Research Fellowship in Science for Meritorious Students (RFSMSs) fellowship to the first author.

\section{References}

[1] M. Galisteo, M. Sánchez, R. Vera et al., "A diet supplemented with husks of Plantago ovata reduces the development of endothelial dysfunction, hypertension, and obesity by affecting adiponectin and TNF- $\alpha$ in Zucker rats," Journal of Nutrition, vol. 135, no. 10, pp. 2399-2404, 2005.

[2] J. W. Anderson, L. D. Allgood, J. Turner, P. R. Oeltgen, and B. P. Daggy, "Effects of psyllium on glucose and serum lipid responses in men with type 2 diabetes and hypercholesterolemia," American Journal of Clinical Nutrition, vol. 70, no. 4, pp. 466-473, 1999.

[3] A. L. Romero, K. L. West, T. Zern, and M. L. Fernandez, "The seeds from Plantago ovata lower plasma lipids by altering hepatic and bile acid metabolism in guinea pigs," Journal of Nutrition, vol. 132, no. 6, pp. 1194-1198, 2002.

[4] J. M. A. Hannan, L. Ali, J. Khaleque, M. Akhter, P. R. Flatt, and Y. H. A. Abdel-Wahab, "Aqueous extracts of husks of Plantago ovata reduce hyperglycaemia in type 1 and type 2 diabetes by inhibition of intestinal glucose absorption," British Journal of Nutrition, vol. 96, no. 1, pp. 131-137, 2006.

[5] L. Lombardi and L. Sebastiani, "Copper toxicity in Prunus cerasifera: growth and antioxidant enzymes responses of in vitro grown plants," Plant Science, vol. 168, no. 3, pp. 797-802, 2005.

[6] H. Panou-Filotheou, A. M. Bosabalidis, and S. Karataglis, "Effects of copper toxicity on leaves of oregano (Origanum vulgare subsp. hirtum)," Annals of Botany, vol. 88, no. 2, pp. 207214, 2001.

[7] I. Yruela, J. J. Pueyo, P. J. Alonso, and R. Picorel, "Photoinhibition of photosystem II from higher plants: effect of copper inhibition," Journal of Biological Chemistry, vol. 271, no. 44, pp. 27408-27415, 1996.

[8] E. Ptsikk, E. M. Aro, and E. Tyystjarvi, "Increase in the quantum yield of photoinhibition contributes to copper toxicity in vivo," Plant Physiology, vol. 117, pp. 619-627, 1998.

[9] T. S. Babu, J. B. Marder, S. Tripuranthakam, D. G. Dixon, and B. M. Greenberg, "Synergistic effects of a photooxidized polycyclic aromatic hydrocarbon and copper on photosynthesis and plant growth: evidence that in vivo formation of reactive oxygen species is a mechanism of copper toxicity," Environmental Toxicology and Chemistry, vol. 20, no. 6, pp. 1351-1358, 2001.

[10] A. R. Sheldon and N. W. Menzies, "The effect of copper toxicity on the growth and root morphology of Rhodes grass (Chloris gayana Knuth.) in resin buffered solution culture," Plant and Soil, vol. 278, no. 1-2, pp. 341-349, 2005.

[11] S. Clemens, "Molecular mechanisms of plant metal tolerance and homeostasis," Planta, vol. 212, pp. 475-486, 2011.

[12] C. Cobbett and P. Goldsbrough, "Phytochelatins and metallothioneins: roles in heavy metal detoxification and homeostasis," Annual Review of Plant Biology, vol. 53, pp. 159-182, 2002.

[13] N. A. L. M. Van Hoof, V. H. Hassinen, H. W. J. Hakvoort et al., "Enhanced copper tolerance in Silene vulgaris (Moench) Garcke populations from copper mines is associated with increased transcript levels of a 2b-type metallothionein gene," Plant Physiology, vol. 126, no. 4, pp. 1519-1526, 2001.

[14] L. Lanfranco, A. Bolchi, E. C. Ros, S. Ottonello, and P. Bonfante, "Differential expression of a metallothionein gene during the presymbiotic versus the symbiotic phase of an arbuscular mycorrhizal fungus," Plant Physiology, vol. 130, no. 1, pp. 58-67, 2002.

[15] K. Bilecen, U. H. Ozturk, A. D. Duru et al., "Triticum durum metallothionein: isolation of the gene and structural characterization of the protein using solution scattering and molecular modeling," Journal of Biological Chemistry, vol. 280, no. 14, pp. 13701-13711, 2005.

[16] G. Mir, J. Domènech, G. Huguet et al., "A plant type 2 metallothionein (MT) from cork tissue responds to oxidative stress," Journal of Experimental Botany, vol. 55, no. 408, pp. 2483-2493, 2004.

[17] M. Das and S. Sen Raychaudhuri, "Enhanced development of somatic embryos of Plantago ovata forsk. By additives," In Vitro Cellular and Developmental Biology, vol. 37, no. 5, pp. 568-571, 2001.

[18] A. Murphy and L. Taiz, "Comparison of metallothionein gene expression and nonprotein thiols in ten Arabidopsis ecotypes. Correlation with copper tolerance," Plant Physiology, vol. 109, no. 3, pp. 945-954, 1995.

[19] K. Edwards, C. Johnstone, and C. Thompson, "A simple and rapid method for the preparation of plant genomic DNA for PCR analysis," Nucleic Acids Research, vol. 19, no. 6, p. 1349, 1991.

[20] M. T. Araya, A. Siah, D. Mateo et al., "Selection and evaluation of housekeeping genes for haemocytes of soft-shell clams (Mya arenaria) challenged with Vibrio splendidus," Journal of Invertebrate Pathology, vol. 99, no. 3, pp. 326-331, 2008.

[21] S. Castiglione, C. Franchin, T. Fossati, G. Lingua, P. Torrigiani, and S. Biondi, "High zinc concentrations reduce rooting capacity and alter metallothionein gene expression in white poplar 
(Populus alba L. cv. Villafranca)," Chemosphere, vol. 67, no. 6, pp. 1117-1126, 2007.

[22] S. -L. The, J. O. Abdullah, and P. Namasivayam, "Molecular cloning, sequencing, and characterization of a putative acetylCoA-C-acetyltransferase cDNA from a highly fragrant orchid hybrid Vanda Mimi Palmer," Sequencing, vol. 2012, Article ID 509034, 8 pages, 2012.

[23] M. S. Krasnikova, I. A. Milyutina, V. K. Bobrova, A. V. Troitsky, A. G. Solovyev, and S. Y. Morozov, "Molecular diversity of miR390-guided transacting siRNA precursor genes in lower land plants: experimental approach and bioinformatics analysis," Sequencing, vol. 2011, Article ID 703683, 6 pages, 2011.

[24] P.-A. Binz and J. H. R. Kagi, "Metallothionein: molecular evolution and classification," in Metallothionein IV, C. Klaassen, Ed., pp. 7-13, Birkhauser, Basel, Switzerland, 1999.

[25] I. El Ghazi, B. L. Martin, and I. M. Armitage, "Metallothionein3 is a component of a multiprotein complex in the mouse brain," Experimental Biology and Medicine, vol. 231, no. 9, pp. 15001506,2006

[26] K. J. Livak and T. D. Schmittgen, "Analysis of relative gene expression data using real-time quantitative PCR and the $2^{-\Delta \Delta \mathrm{CT}}$ method," Methods, vol. 25, no. 4, pp. 402-408, 2001.

[27] U. Pieper, B. M. Webb, D. T. Barkan et al., "MODBASE, a database of annotated comparative protein structure models and associated resources," Nucleic Acids Research, vol. 39, pp. 465-474, 2011.

[28] N. Eswar, B. John, N. Mirkovic et al., "Tools for comparative protein structure modeling and analysis," Nucleic Acids Research, vol. 31, no. 13, pp. 3375-3380, 2003.

[29] J. Zhou and P. B. Goldsbrough, "Structure, organization and expression of the metallothionein gene family in Arabidopsis," Molecular and General Genetics, vol. 248, no. 3, pp. 318-328, 1995.

[30] S. H. Cho, Q. T. Hoang, Y. Y. Kim et al., "Proteome analysis of gametophores identified a metallothionein involved in various abiotic stress responses in Physcomitrella patens," Plant Cell Reports, vol. 25, no. 5, pp. 475-488, 2006.

[31] G.-Y. Huang and Y.-S. Wang, "Expression analysis of type 2 metallothionein gene in mangrove species (Bruguiera gymnorrhiza) under heavy metal stress," Chemosphere, vol. 77, no. 7, pp. 10261029, 2009.

[32] A. Macovei, L. Ventura, M. Donà, M. Faè, A. Balestrazzi, and D. Carbonera, "Effect of heavy metal treatments on metallothionein expression profiles in white poplar (Populus alba L.) cell suspension cultures," Analele Universitatii Din Oradea-Fascicula Biologie, vol. 27, no. 2, pp. 274-279, 2010. 


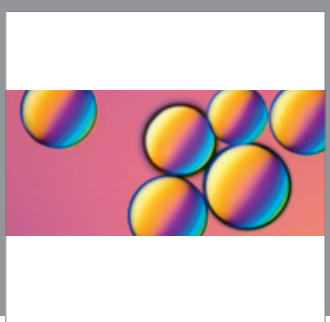

Peptides

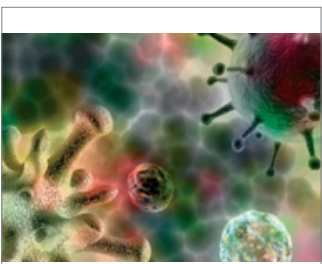

\section{Virology}

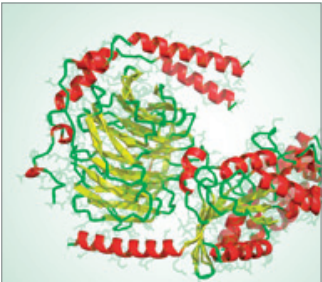

Signal Transduction
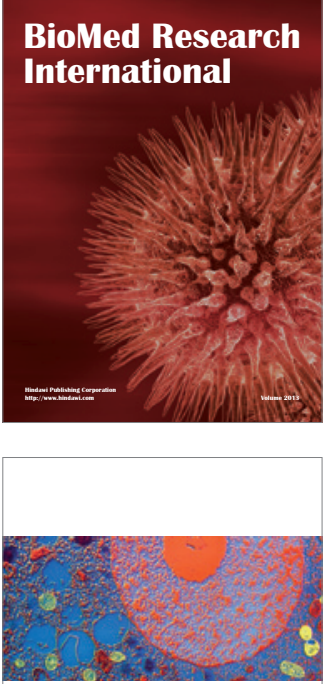

ISRN

Cell Biology
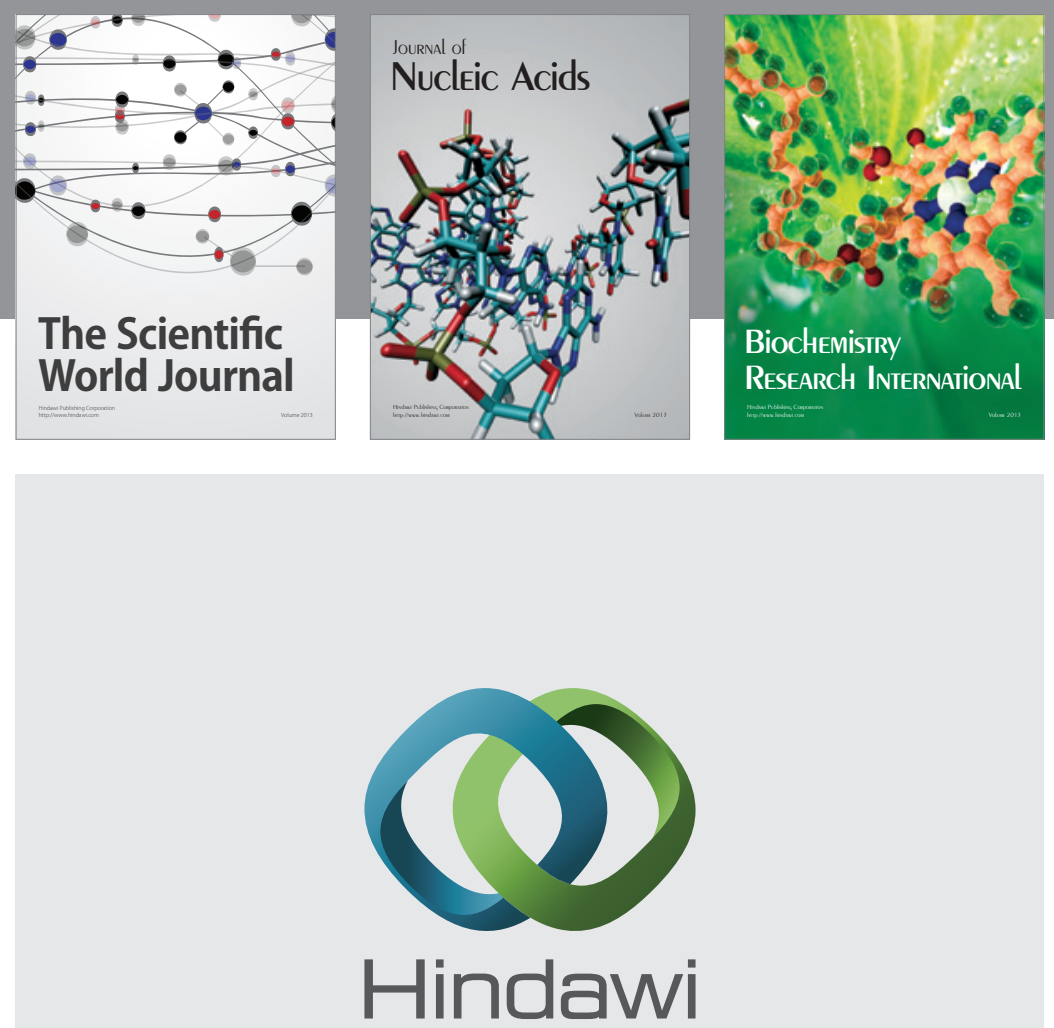

Submit your manuscripts at http://www.hindawi.com

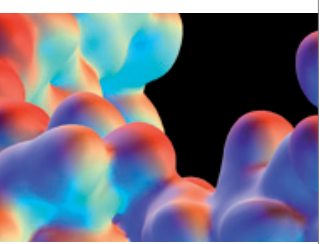

Enzyme Research

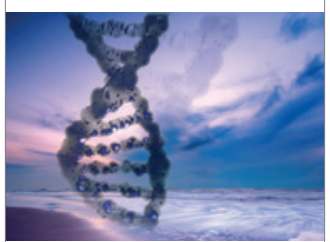

Evolutionary Biology
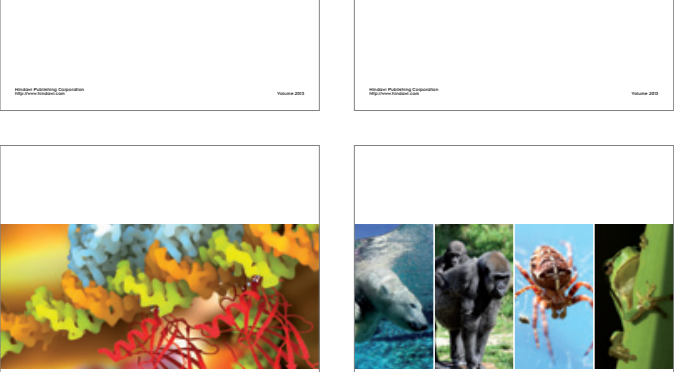

ISRN

Molecular Biology

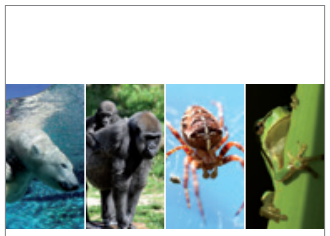

ISRN Zoology

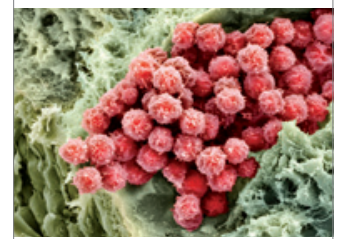

Stem Cells International

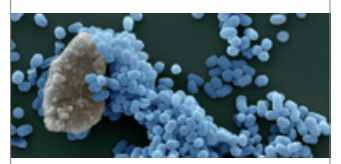

ISRN

Biotechnology
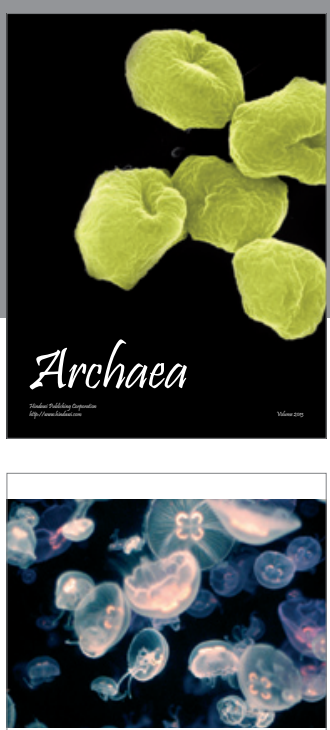

Journal of Marine Biology
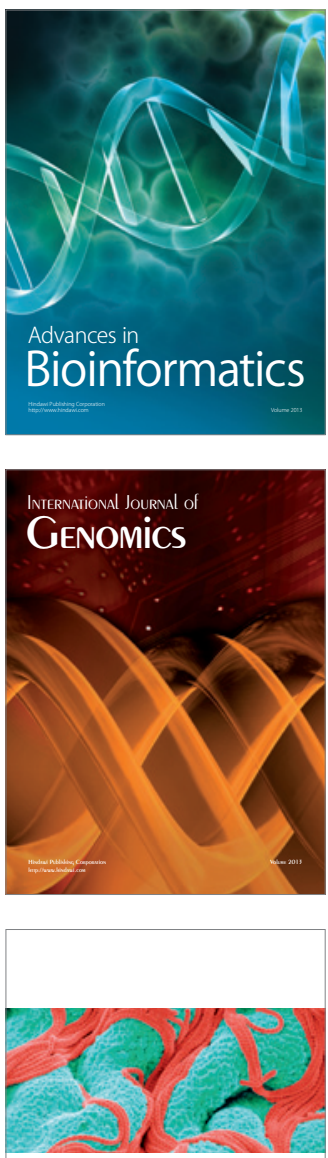

ISRN

Microbiology 